\title{
Si comincia dal cappone: vent'anni di Presìdi Slow Food
}

All started with a capon: 20 Years of Slow Food Presidia

Davide Porporato

\section{OpenEdition}

Journals

Edizione digitale

URL: http://journals.openedition.org/aam/309

DOI: 10.4000/aam.309

ISSN: 2038-3215

Editore

Dipartimento Culture e Società - Università di Palermo

Notizia bibliografica digitale

Davide Porporato, «Si comincia dal cappone: vent'anni di Presidi Slow Food », Archivio antropologico mediterraneo [Online], Anno XXI, n. 20 (1) | 2018, online dal 30 juin 2018, consultato il 28 décembre 2019. URL : http://journals.openedition.org/aam/309; DOI : 10.4000/aam.309

Questo documento è stato generato automaticamente il 28 dicembre 2019

Archivio antropologico mediterraneo 


\title{
Si comincia dal cappone: vent'anni di Presìdi Slow Food
}

\author{
All started with a capon: 20 Years of Slow Food Presidia
}

Davide Porporato

1 1. Negli ultimi anni il vasto tema della biodiversità ha conosciuto un crescente interesse in ambiti scientifici differenti. Sono state prodotte ricerche che concordano nell'evidenziare come l'impatto antropico sulla diversità delle specie animali e vegetali, avviato dalla rivoluzione industriale e ampliato dalla globalizzazione economica, sia correlato a un significativo e preoccupante depauperamento della biodiversità: un dato riscontrabile, con percentuali differenti, in ogni parte della Terra (Raisson 2010: 146-147; Maffi, Woodley 2010; Bevilacqua 2006). Le molteplici ragioni del declino sono riconducibili a un insieme di cause tra loro combinate, fra le quali la modifica e distruzione degli habitat, l'inquinamento, il cambiamento climatico (Crutzen 2002; 2005); ma anche l'adozione di sistemi di produzione alimentare che nel privilegiare, per ragioni economiche, la coltivazione e l'allevamento di un numero ristretto di varietà vegetali e animali ha contribuito, di fatto, a rendere gli ecosistemi più fragili, più vulnerabili ${ }^{1}$. Una riduzione e una semplificazione facilmente riscontrabili in molti territori, e che hanno impoverito, in modo rilevante, la biodiversità che ancora segnava, ad esempio, l'Italia preindustriale.

2 La contemporaneità è caratterizzata da un sistema agroalimentare transplanetario, che tende sempre più a imporre modelli di produzione e di consumo fondati sul principio in base al quale l'agricoltura locale deve essere al servizio del mercato globale. Tutto ciò avviene senza un'adeguata valutazione delle ricadute sull'ambiente e sulla vita delle comunità. Tali politiche hanno favorito, e talvolta imposto, le colture da reddito a scapito di quelle per la sussistenza:

in questo modo il cibo è stato trasformato in merce, senza prestare attenzione alle implicazioni culturali e sociali che questa trasformazione ha comportato, impoverendo ogni altro sistema di valori e conoscenza basato sulla ricerca del benessere attraverso un approccio più olistico (Bravo et al. 2011: 19). 

in molti casi, la dimenticanza, persino, dei tratti costitutivi del tempo calendariale tradizionale: una trama simbolica complessa che permetteva all'uomo di orientarsi e di organizzarsi nella società lungo l'asse della propria esistenza annuale e di vita (Grimaldi 1993; Buttitta 2013).

4 In questo scenario sono nate e si sono consolidate iniziative che propongono l'adozione di innovativi sistemi di governance delle produzioni locali accomunate dal fatto di ritenere fondamentale la salvaguardia della biodiversità. Una minore attenzione è stata invece riservata alla tutela, alla difesa delle pratiche tradizionali associate ai cicli produttivi. Un insieme di saperi, di natura orale e gestuale, che nel corso del tempo si sono sedimentati nei differenti contesti, contribuendo a definire i marcatori e i simboli delle identità locali. Ogni gruppo umano ha infatti prodotto nel corso della storia strategie materiali e immateriali per procurarsi le risorse necessarie alla sopravvivenza, per favorire e proteggere la vita, per renderla sostenibile dai propri membri nei differenti contesti ambientali. Si tratta in genere di conoscenze empiriche, esito di una ricercata e attenta mediazione tra uomo e natura, che connotano le comunità locali e rappresentano un patrimonio di etnodiversità inteso come

preziosa eredità locale che differenzia ogni gruppo umano e che tuttavia, sia ben chiaro, non si realizza nell'isolamento e nella xenofobia, ma nello scambio e nell'intreccio fra tradizioni e culture. In altre parole, l'etnodiversità consiste nel riconoscere, abbracciare e, nel contempo, sottolineare le diversità, le specificità linguistiche, le tradizioni, i saperi, i gesti e le parole [...] che costituiscono i precari, ma nel contempo solidi, mattoncini costitutivi della memoria orale del mondo (Bravo et al. 2011: 19).

5 2. Tra le iniziative che hanno avuto il merito di considerare la dimensione della biodiversità senza disgiungerla da quella dell'etnodiversità, vedendole come due facce della stessa medaglia, come due categorie che necessariamente devono tra loro dialogare criticamente per ripensare le pratiche della produzione e del consumo del cibo, troviamo il progetto Presìdi², avviato alla fine degli anni Novanta dall'Associazione internazionale Slow Food ${ }^{3}$ all'interno di un più vasto progetto denominato Arca del Gusto (Milano et al. 2015) ${ }^{4}$, nato nel 1996 con l'obiettivo di condurre un primo censimento dei prodotti agroalimentari in pericolo di estinzione. L'impianto progettuale venne ridiscusso nel 1998, in occasione della seconda edizione del ‘Salone del Gusto' di Torino, perché occorreva andare oltre la mera catalogazione:

Si concretizzò così l'idea del Presidio, cioè di un intervento diretto che coinvolgesse i produttori e le realtà di supporto locali [...] e che via via si amplificasse sino a comporre una rete su tutto il territorio nazionale in grado di tutelare e valorizzare razze autoctone, formaggi, salumi, legumi, ortaggi, dolci e frutti legati a un territorio da un punto di vista ambientale, storico e socioeconomico (Battaglio, Sardo 2010: 17).

6 I Presìdi sono dunque un'iniziativa che cerca di contrastare la perdita della bio ed etno diversità, ma anche lo spopolamento delle aree interne del mondo attraverso un insieme di interventi virtuosi volti a creare le condizioni per una più organica valorizzazione delle produzioni: un requisito indispensabile per superare l'isolamento e lo svuotamento nel quale versano molti territori non solo in Italia ${ }^{5}$. Nel 2018 risultano attivi 537 Presìdi in oltre sessanta nazioni, di questi 293 sono in Italia e coinvolgono 
oltre duemila piccoli produttori. Si tratta di realtà che sperimentano un nuovo modo, sistemico e creativo, di pensare e affrontare i temi della produzione alimentare, fondato sul legame con il territorio, sul recupero dei saperi tradizionali, sulla piccola scala, sulla sostenibilità ambientale e sociale e sul benessere animale. Azioni che tendono a sviluppare una forte alleanza tra chi il cibo lo produce e chi lo consuma, riconoscendone il valore socioculturale, ambientale ed economico (Peano, Sottile 2012: 3-5). I Presìdi sono esperienze concrete, ampiamente sperimentate da due decenni in vari luoghi della Terra, che consentono di riconsiderare e ripensare il cibo non come una merce come tutte le altre e di scongiurare, in questo modo, il pericolo, opportunamente evidenziato da Carlo Petrini con una formula icastica, di «essere mangiati dal cibo» (Petrini 2009).

7 Nel corso del 2012, nell'ambito di un progetto cofinanziato dalla Commissione Europea, la Fondazione Slow Food per la Biodiversità ha avviato con le Università di Torino e di Palermo un'interessante ricerca scientifica quali-quantitativa allo scopo di elaborare un modello di valutazione della sostenibilità dei Presìdi, declinata nella triplice dimensioni socioculturale, ambientale ed economica ${ }^{6}$. L'indagine, che ha interessato un campione di diciassette Presìdi europei nati tra il 2000 e il 2009, ha evidenziato un dato complessivo particolarmente rilevante. Si registra infatti un significativo aumento della sostenibilità socioculturale dovuta a una maggiore interazione tra i produttori, unita a una crescente consapevolezza del valore del proprio lavoro e del prodotto, esito a sua volta di una più efficace comunicazione e partecipazione a eventi pubblici. Significative in questo ambito sono anche le associazioni di produttori nate per meglio organizzare il lavoro del presidio e aumentarne il potere contrattuale. Anche la sostenibilità ambientale ne trae vantaggio: i dati raccolti mostrano infatti una tendenza a ridurre l'uso di concimi di sintesi a favore di quelli organici di origine naturale. Pure nell'alimentazione degli animali si fa un uso minore, o addirittura si fa a meno, degli insilati e dei mangimi contenenti organismi geneticamente modificati. In questo quadro di cambiamento positivo risultano ancora carenti le iniziative indirizzate all'uso di energie alternative e di materiali per il packaging a basso impatto ambientale. L'ultimo parametro, quello della sostenibilità economica, evidenzia un quadro particolarmente positivo in ogni realtà indagata (Peano, Sottile 2012: 17-18; Peano et al. 2014). Nel corso del 2013 una nuova ricerca, che ha interessato 44 Presìdi di montagna attivi sulle Alpi, gli Appennini, i Pirenei, i Carpazi e il Caucaso, ha confermato il quadro già emerso nella prima rilevazione: vale a dire un netto miglioramento nella scala di sostenibilità totale, con una crescita rilevante della dimensione socioculturale (Battaglino et al. 2014: 38-52; Fassino 2015).

8 Occorre ancora soffermarsi su un'altra iniziativa che coinvolge i produttori dei Presidi. Dal 2013 alcuni prodotti si presentano al pubblico con una 'etichetta narrante' che, accanto a quella prevista dalle normative, fornisce all'acquirente informazioni sul territorio, sull'azienda, sul produttore e sulle tecniche di coltivazione e di allevamento (Baldereschi et al. 2014). Un progetto comunicativo col quale si tende a superare il tecnicismo che accompagna l'etichetta legale per offrire l'opportunità di far conoscere, almeno in parte, quel vasto patrimonio di conoscenze, spesso di natura orale e gestuale, che frequentemente si nascondono dietro al cibo: l'origine, la storia, le tecniche di lavorazione, informando nello stesso tempo se esso è prodotto nel rispetto del buono, pulito, giusto, aspetti interconnessi di un'unitaria idea olistica di umanità, che sta a fondamento del pensiero di Slow Food (Petrini 2005b; 2012). Questo percorso 
conoscitivo e pedagogico ha radici antiche: già duemila anni fa Plinio il Vecchio scriveva nella Naturalis Historia: "Cominceremo ora a trattare l'opera più grandiosa della natura: esporremo all'uomo i suoi cibi, e lo costringeremo ad ammettere che gli è sconosciuto ciò che lo fa vivere» (Plinio 1982-1988: XX,1).

9 Le etichette di cui parliamo possono inoltre essere dotate di un $Q R$ Code (Quick Response Code) o di una smart label ${ }^{7}$ il che segna un radicale cambiamento di status: l'etichetta infatti diviene un oggetto digitale capace di narrare il prodotto al quale è associata, facendo ricorso a contenuti che non sono presenti all'interno dell'hortus conclusus definito dal perimetro analogico del supporto cartaceo. Ciò permette di associare alla materialità del prodotto l'immaterialità di contenuti digitali e multimediali, interconnessi e interattivi, depositati online. Si creano così le condizioni per veicolare saperi etnoantropologici riferiti a specifici areali, di comunicare al cliente quello che i francesi chiamano terroir, un termine difficilmente traducibile, che a partire dall'Ottocento viene utilizzato per definire un territorio attraverso una costellazione concettuale polisemica, capace di comprendere natura e cultura e di far dialogare la dimensione materiale con quella immateriale (Porporato 2016: 196-197). In altre parole, l'etichetta narrante contribuisce anche a definire il concetto di 'cibo geografico' che, come è noto, rappresenta un tratto peculiare della dimensione gastronomica italiana fondata sulle specificità territoriali (Montanari 2006), e consente al gesto e alla parola di correre, come afferma Piercarlo Grimaldi, «nello spazio e nel tempo digitali dando vita a un'enciclopedia dei saperi per molti versi labile e immateriale, forse più di quanto lo fosse la memoria orale del passato» (Grimaldi, Nattino 2009: 9-10). L'hortus conclusus dell'etichetta tradizionale si è trasformato in un crocevia numerico a partire dal quale si può interagire con l'illimitato universo delle relazioni digitali che costituiscono il nuovo mondo smaterializzato, ampio quanto il mondo, e nello stesso tempo 'liquido': i contenuti, i contesti e le relazioni, sempre più disincarnate, alle quali esso rinvia, si ridefiniscono e si modificano rapidamente (Bauman 2008). A tale proposito si può condividere l'opinione di Matilde Callari Galli, secondo la quale «La comunità che diviene il campo del lavoro antropologico non è più stabilita da confini geografici e/o amministrativi - il comune del Mezzogiorno italiano o l'isola dei Mari del Sud, le riserve indiane o il "popolo della Sierra" -; piuttosto è costituita da individui legati da vincoli definiti soprattutto dal vivere più o meno stabilmente esperienze comuni, dal condividere per un certo lasso di tempo situazioni rilevanti per gli obiettivi che la ricerca etnografica si pone» (Callari Galli 2007: 23).

10 3. A partire da queste considerazioni abbiamo verificato sul campo il quadro finora evidenziato, indagando il primo presidio costituito da Slow Food, quello del Cappone di Morozzo, piccolo comune della campagna cuneese non lontano dal capoluogo di provincia e da Mondovì.

11 Nell'ultimo quarto del Novecento questa produzione tradizionale si era fortemente contratta e i galletti castrati chirurgicamente e allevati dalle donne contadine del luogo erano rimasti poche centinaia. Anche l'annuale 'Fiera del cappone', che si svolge nella seconda domenica e lunedì di dicembre, appariva ormai in via di dismissione. Carlo Petrini osserva che nel 1998 
il panorama della fiera era davvero avvilente: essendo da queste parti profondamente mutata l'economia contadina, la tradizione del cappone si stava perdendo, poche donne lo allevavano ancora e ancora meno erano i compratori in un momento che sarebbe dovuto essere di festa. In sostanza, la pratica onerosa di allevare queste bestie, animata da grande savoir faire e passione, non era più gratificata da una giusta remunerazione e aveva ridotto la fiera a una testimonianza molto triste sui tempi che furono ${ }^{9}$ (Petrini 2005b: 162-163). pratica diffusa su tutto il territorio italiano e in numerosi paesi europei, e trova numerose attestazioni nel corso della storia. Per il mondo antico forniscono interessanti e curiose descrizioni sulle modalità dell'intervento di capponatura Aristotele, Terenzio Varrone, Plinio il Vecchio e Columella. Quest'ultimo, tra gli antichi, è il primo a parlare di asportazione degli organi genitali, una pratica necessaria che segna il passaggio dal gallo al cappone: «Questo è il nome che si dà ad essi, quando si sono castrati per abolirne la libidine. Né questo succede solo se si tolgono gli organi genitali, ma anche se si bruciano gli speroni con un ferro incandescente e, quando il fuoco li ha consumati, le piaghe prodotte si spalmano di creta da vasaio» (1992: 562-563). Gli altri autori insistono sulla bruciatura della cloaca e degli speroni. Nei primi anni del Seicento l'agronomo e botanico francese Olivier de Serres (1605: 326-327) riferisce che la castrazione dei galli era compito esclusivo delle contadine, così come confermava nella prima parte dell'Ottocento Hurtrel D'Arboval, nel Dizionario di medicina chirurgia ed igiene veterinaria (1839: 457). Le testimonianze raccolte sul campo hanno evidenziato come la capponatura sia rimasta, ancora per tutto il Novecento, una pratica esclusivamente femminile, un sapere trasmesso da nonna a nipote, da madre a figlia $^{10}$.

dunque le donne contadine le eredi e depositarie di questo sapere antico, una pratica che, mentre toglie aggiunge qualità e quantità all'animale. Un intervento che fino a pochi anni fa risultava particolarmente cruento: si cominciava con un piccolo taglio nell'addome che permetteva l'asportazione dei testicoli, operazione cui seguiva la recisione della cresta, dei bargigli e talvolta degli speroni che in alcuni casi venivano innestati nella ferita lasciata dall'asportazione della cresta, simulando in un certo senso la mascolinità di cui i galli erano stati privati, e quasi a titolo di ricompensa per il vulnus irrimediabilmente inferto ${ }^{11}$. Le interviste realizzate ci dicono anche che il delicato intervento doveva essere preferibilmente eseguito all'inizio dell'estate, con la luna calante e nelle ore più fresche della giornata, all'alba o al crepuscolo. La ferita di pochi centimetri, dopo esser stata cucita con ago e filo, veniva disinfettata con acqua, grappa, aceto e alle volte cosparsa di cenere. Si registra anche l'usanza di far inghiottire i testicoli allo stesso animale appena evirato, oppure di cucinarli per i ragazzi di casa per favorirne la virilità. Di contro questo uso alimentare è sconsigliato alle donne poiché «ne risentirebbero nocumento: col fatto che le loro mani sarebbero soggette al sudore, tanto da essere malagevoli i lavori, e in particolare il trar l'ago» (Baldini 2003: 69).

In Piemonte l'uso gastronomico delle parti sottratte al galletto costituiscono gli ingredienti base di un piatto povero molto diffuso in tutta la regione: la finanziera. Per 
comprendere l'origine di questo nome è opportuno considerare, come sostiene Gian Luigi Beccaria, che

le parole che usiamo sono lo specchio di vicende accadute, e di consuetudini. [...] la finanziera: si dice sia nato nel sec. XIX per accontentare le esigenze di frettolosi uomini d'affari i quali volevano consumare un pasto rapido. Da loro deriverebbe il nome del cibo, o meglio dalla «finanziera», il tipo di giacca che nell'ottocento indossavano i finanzieri e gli uomini d'affare. Può darsi però che la storia sia un'altra, perché si tratta di un piatto povero, poco adatto per gente di rango, un piatto composto di recuperi: quando si castravano i capponi, creste bargigli granelli erano disponibili, bastava aggiungere fegatini di pollo, animelle, e il piatto era pressoché completo, e allora dovremmo piuttosto pensare al tributo che i contadini che venivano in città a vendere il pollame pagavano ai finanzieri, regalando loro fegato, cuore, ventrigli, granelli, creste, bargigli (2007: 52).

Il gallo castrato perdeva la capacità di cantare, «la sua natura solare, la funzione di nunzio del giorno e della luce che liberava dalle tenebre, e infine il ruolo di capo e difensore del pollaio» (Cattabiani 2000: 218) ${ }^{12}$; abbandonando vigore e aggressività gli si poteva anche affidare la custodia dei pulcini appena schiusi o addirittura la cova delle uova, sostituendo in tale compito la gallina, che poteva così proseguire a deporre le uova. La ricerca di terreno ha documentato, inoltre, le pratiche adottate per 'addestrare' il cappone alla cova e alla conduzione dei pulcini ${ }^{13}$.

Il cappone non svolge dunque una funzione soltanto passiva, di animale da ingrasso, allevato all'unico scopo di ottenere una carne di qualità superiore, ma collabora attivamente all'economia dell'azienda agricola. Ciò fa capire come i cicli di quest'ultima prefigurino le logiche della produzione su larga scala, diversi e successivi ma non del tutto dissimili dall'organizzazione del lavoro che li ha preceduti. I saperi dell'oralità indicano particolarmente adatti a svolgere il ruolo di chioccia i galletti capponati nel giorno di san Rocco "perché solo così acquistano la facoltà di emettere quel caratteristico croc croc proprio della chioccia nel chiamare a sé i pulcini. (Forse per ragioni di onomatopeia: Rocco $=$ croc!)» (Cavazzutti 1933: 45). Lo stesso filo di lino o canapa utilizzato per chiudere la ferita assumeva un potere magico e portava fortuna a colui che se lo trovava tra i denti durante il pranzo di Natale oppure al giocatore di carte al quale era stato messo in una tasca a sua insaputa (Baccocco 1893: 237).

Grazie alla particolare bontà i capponi erano il dono natalizio più atteso, offerto al proprietario del fondo, al notabile locale, al dottore, alla levatrice e al maestro di scuola; costituivano il piatto forte del pranzo di Natale (Zucca 1992: 110). Ma erano regalati anche in altre feste del ciclo annuale e della vita (Maragliano 1962: 53-54). Va ancora osservato che il loro consumo, in alcuni territori italiani, segna anche la cucina grassa del tempo carnevalesco come opportunamente osserva Giovanni Ciappelli:

Tipici di questa particolare occasione dell'anno, e in particolare del pranzo dell'ultimo giorno di Carnevale, siano i capponi, al punto che un fiorentino doveva ritenere che i festeggiamenti di Carnevale non fossero degni di questo nome se uno o più dei grassi volatili non erano presenti sulla tavola (1997: 75).

Significativo il passaggio di manzoniana memoria nel quale Agnese suggerisce a Renzo di non recarsi a mani vuote dall'avvocato Azzeccagarbugli ma di portare in dono ben quattro capponi: «Pigliate quei quattro capponi, poveretti! a cui dovevo tirare il collo, per il banchetto di domenica, e portateglieli; perché non bisogna mai andar con le mani vote da que' signori» (Manzoni 1962: 51). In terra di Romagna la comare aveva l'obbligo, nel giorno del battesimo, di portare con sé due capponi mentre la madre della sposa, che partoriva per la prima volta, contribuiva al banchetto che si svolgeva otto giorni 
dopo il parto, con quattro capponi se il neonato era maschio e due se femmina (Cavazzutti 1933: 38). A Pagani, comune della provincia di Salerno, nella prima domenica dopo Pasqua si svolge la festa della Madonna del Carmelo detta delle Galline. Durante la processione vengono offerti alla Madonna, lanciati da finestre e balconi, numerosi volatili tra cui capponi (Rivera 1988: 355 ).

20 È in questa cornice, che offre solo un cenno della vasta e articolata dimensione culturale, sociale ed economica associata alla pratica tradizionale della capponatura, che nasce, tra il 1998 e il 1999, il Presidio Slow Food di Morozzo. Il primo intervento, tutto orientato a salvare il salvabile, è consistito nel convincere le contadine ad aumentare la produzione garantendo loro una remunerazione superiore. Carlo Petrini scrive: «Pensai di proporre alle allevatrici di preparare mille capponi per l'anno successivo che mi sarei impegnato a comprare per un prezzo quasi doppio di quello che veniva pagato nel '98» (2005b: 163). L'azione commerciale, andata a buon fine, ha avuto il merito di ridare fiducia ai pochi produttori allora attivi e nel contempo ha dimostrato che il cappone di Morozzo poteva tornare a essere un cibo «buono da mangiare, buono da pensare» (Lévi-Strauss 1962).

21 Un passaggio rilevante avviene nel 2001 con l'istituzione del 'Consorzio per la tutela e la valorizzazione del cappone di Morozzo e delle produzioni avicole tradizionali' che oggi riunisce una quarantina di allevatori distribuiti fra tredici comuni limitrofi a Morozzo. Le aziende agricole operano nel rispetto di un rigido disciplinare che impegna gli associati ad allevare la 'razza nostrana - biotipo scuro di Cuneo', conosciuta anche come 'Nostrale di Morozzo', stabilisce il tempo minimo di vita dell'animale e i giorni che devono trascorrere tra l'intervento di orchiectomia e la macellazione, ma soprattutto definisce le modalità di allevamento a terra in ampie superfici, e l'alimentazione, che deve essere solo di tipo vegetale a base di granoturco e vieta l'uso di antibiotici e di fattori migliorativi della crescita.

22 Da qualche anno l'intervento di castrazione è radicalmente mutato; la tecnica ora adottata consente l'asportazione dei testicoli attraverso un piccolo taglio nel costato, che si rimargina spontaneamente senza la necessità di sutura chirurgica e, nel rispetto delle normative sul benessere animale, non vengono più recisi cresta, bargigli e speroni che, peraltro, sono ininfluenti sulla castrazione ${ }^{14}$. Con la maturità il cappone raggiunge un peso che varia tra i due e i tre chilogrammi, pelle gialla e un piumaggio di colore intenso e variopinto. Oggi sono circa seimila i galletti che, dopo la capponatura primaverile, vengono venduti a fine anno, in occasione delle festività natalizie.

Il rilancio della produzione è andato di pari passo con la ripresa della storica fiera prenatalizia. Oggi, nell'occasione festiva di metà dicembre, la piazza si riempie di capponi in mostra, un paio per gabbia per rendere evidente l'assenza di aggressività e di impeto dei volatili, quale prova certa dell'avvenuta trasformazione del gallo in cappone (Foto 1, 2). Gli animali in fiera sono affiancati dalle donne e dagli uomini che li hanno allevati e che per l'occasione si trasformano in abili narratori capaci di raccontare a una molteplicità di clienti (commercianti, macellai, ristoratori e privati provenienti da tutta Italia) e con dovizia di particolari, la lunga storia del cappone, il ciclo produttivo tradizionale e contemporaneo. Nell'occasione il frutto di un anno di lavoro è offerto al pubblico con un orgoglio ritrovato, il cappone è ora un rilevante attrattore economico, sociale e culturale per il territorio, e il tempo in cui più nessuno lo cercava appare lontano. Poter dire di aver acquistato un cappone di Morozzo è motivo di vanto, l'efficace valorizzazione operata è comprovata anche da una 
accresciuta presenza nell'immaginario che ha reso il cappone e la sua fiera un riferimento, un indice di misura, un parametro di valutazione adottato per valutare il successo di altre analoghe esperienze che rispondono alla stessa logica di patrimonializzazione: la quale, va ricordato, ha saputo far interagire la dimensione materiale con quella immateriale. Va ancora segnalato che la fiera offre anche l'occasione per conoscere altri prodotti del territorio, soprattutto vini e formaggi.

Il Presidio è inoltre diventato luogo di stage per gli studenti dell'Università di Scienze Gastronomiche di Pollenzo. Una destinazione appena fuori porta, che consente di integrare e completare percorsi conoscitivi avviati in aula e di sperimentare una didattica esperienziale ritenuta fondamentale per la comprensione delle scienze gastronomiche. Trascorrere del tempo a indagare il sistema di sussistenza di una comunità africana o la funzione di un Presidio Slow Food in provincia di Cuneo sono esperienze altrettanto significative perché, come sostiene Carlo Petrini,

rappresentano il modo più diretto per dare dignità accademica ai saperi dei contadini, degli artigiani, dei produttori, degli allevatori, dei distributori sostenibili. Un modo per farli diventare docenti, come meritano. È quella realizzazione di un 'dialogo tra regni' che auspico almeno da una decina d'anni, lavorando perché la scienza ufficiale e accademica si metta sullo stesso piano dei saperi tradizionali del mondo del cibo, e quindi instauri una forma paritaria di dialettica e d'interazione (2013: 45-46).

L'Università, inoltre, incoraggia gli studenti a documentare filmicamente le esperienze vissute, a raccogliere storie di vita che possono essere custodite e mostrate al mondo grazie al progetto 'Granai della memoria', un percorso scientifico e didattico ambizioso che si pone l'obiettivo di salvaguardare i saperi orali e gestuali della tradizione ${ }^{15}$ (Grimaldi, Porporato 2012: 12; Porporato, Fassino 2016: 179-193).

In conclusione abbiamo trattato un caso in cui il rinnovato dialogo tra natura e cultura è a fondamento della rinascita di un territorio. La patrimonializzazione della capponatura è diventata parte integrante della vita della comunità, tanto che il cappone è rappresentato da un 'totem', che cattura l'attenzione di chi percorre la strada che conduce a Morozzo (Foto 3).

La ripresa di questo ciclo produttivo è dunque un'innovazione ben riuscita che ha favorito, innescato e disseminato l'iniziativa dei Presìdi. Da questa prima esperienza è partito un messaggio che ora viaggia per il mondo, testimoniando come un armonico uso della biodiversità e dell'etnodiversità possa rappresentare una speranza di futuro per le campagne. In esse, nel contesto di una dirompente crisi economica, «diventano nuovi fattori economici anche l'amicizia, la fiducia, i sentimenti, le relazioni fra le persone, gli scambi, le reciproche opportunità» (Nattino 2010: 260).

Gli ambiti locali, se vogliono sopravvivere all'omologazione culturale generata dai processi di globalizzazione, devono «allearsi creativamente con la tradizione» (Grimaldi 2010: 124): proprio quella tradizione che in precedenza era ritenuta un ostacolo alla marcia inarrestabile del progresso e della modernizzazione, e che ora invece è stata ampiamente rivalutata. Il caso del Presidio di Morozzo che qui abbiamo indagato testimonia e conferma quanto il recupero di una pratica 'tradizionale', come è indubbiamente quella della capponatura, possa rappresentare non già un nostalgico attardarsi verso usi del passato, bensì costituisca oggi un originale ciclo produttivo che, coniugando creativamente passato e presente, trova spazi di mercato che danno sostegno alle comunità locali di produttori. Il fatto che le tecniche di capponatura $\mathrm{e}$ allevamento si siano nel corso degli ultimi anni trasformate, attesta come, anche in 
questo caso, la 'tradizione' non sia una statica ripetizione di gesti, uno stanco perpetuarsi del passato nel presente, bensì un dinamico e incessante processo di reinvenzione. Gérard Lenclud, riprendendo le riflessioni di Jean Pouillon (1975), definisce questo fenomeno «filiazione inversa: non sono i padri a generare i figli, ma i figli che generano i propri padri. Non è il passato a produrre il presente, ma il presente che modella il suo passato. La tradizione è un processo di riconoscimento di paternità» (2001: 131).

\section{BIBLIOGRAFIA}

Andrews G., 2008 The Slow Food Story. Politics and Pleasure, McGill-Queen's University Press, Montreal; trad. it. 2010 Slow food. Una storia tra politica e piacere, Il Mulino, Bologna.

Baccocco M., 1893 «Il Capodanno a Castel Bolognese. Pregiudizi e costumanze», in Rivista delle tradizioni popolari italiane, Anno I: 236-238.

Baldereschi F. et al., 2014 L'etichetta narrante. Una rivoluzione nella comunicazione del cibo, Slow Food, Fondazione Slow Food per la Biodiversità Onlus, Bra.

Baldini E., 2003 La sacra tavola, Bologna, Pendagron, Bologna.

Battaglio D., Sardo S. (a cura di), 2010 Il buon paese. 2500 indirizzi. Il meglio della produzione alimentare italiana, Slow Food Editore, Bra.

Battaglino C. et al. (a cura di), 2014 Bilancio sociale 2013, Fondazione Slow Food per la Biodiversità Onlus, Bra.

Bauman Z., 2008 Vita liquida, Laterza, Roma-Bari.

Beccaria G. L., 2007 Tra le pieghe delle parole. Lingua storia cultura, Einaudi, Torino.

Bevilacqua P., 2006 La Terra è finita. Breve storia dell'ambiente, Laterza, Roma-Bari.

Biroli G., 1812 Trattato di agricoltura, Volume IV, Mezzotti e Vercellotti, Novara.

Bologna G., 2008 Manuale della sostenibilità. Idee, concetti, nuove discipline capaci di futuro, Edizioni Ambiente, Milano.

Bravo G. L. et al., 2011 Conoscenze tradizionali, le questioni di genere e valori immateriali, in Politiche alimentari e sostenibilità, Slow Food Editore, Bra: 18-21.

Bukowski W., 2015 La danza delle mozzarelle. Slow Food, Eataly, Coop e la loro narrazione, Alegre, Roma. Buttitta I., 2013 Continuità delle forme e mutamento dei sensi. Ricerche e analisi sul simbolismo festivo, Bonanno, Acireale-Roma.

Callari Galli M., 2007 «Pratiche etnografiche nella città contemporanea», in M. Callari Galli (a cura di), Mappe urbane. Per un'etnografia della città, Guaraldi, Rimini: 7-41.

Cattabiani A., 2000 Volario, Mondadori, Milano.

Cavazzutti E., 1933 «Usanze di Romagna», in Lares, IV: 38-45. 
Ciappelli G., 1997 Carnevale e Quaresima. Comportamenti sociali e cultura a Firenze nel Rinascimento, Edizioni di storia e Letteratura, Roma.

Columella L. G. M., 1992 L'arte dell'agricoltura e libro sugli alberi, Einaudi, Torino.

Crutzen P. J., 2002 «Geology of mankind», in Nature, vol. 415; 23.

Crutzen P. J., 2005 Benvenuti nell'Antropocene. L'uomo ha cambiato il clima, la Terra entra in una nuova era, Mondadori, Milano.

De Serres O., 1605 Le théâtre d'agriculture et mesnage des champs, Ed. Chés Abr. Saugrain, Paris.

Doglio S., 1995 Il dizionario di gastronomia del Piemonte, Daumerie, San Giorgio di Montiglio.

D’Onofrio S., 1997 Le parole delle cose: simboli e riti sociali in Sicilia, Congedo, Galatina.

Fassino G., 2015 «Presìdi Slow Food e buone pratiche di comunità. Il caso della Piattella canavesana di Cortereggio», in P. Corvo, G. Fassino (a cura di), Quando il cibo è benessere. Alimentazione e qualità della vita, Franco Angeli, Milano: 191-205.

Gera F., 1840 Nuovo dizionario universale di agricoltura, Antonelli, Venezia.

Grimaldi P., 1993 Il calendario rituale contadino. Il tempo della festa e del lavoro fra tradizione e complessità sociale, Franco Angeli, Milano.

Grimaldi P., 2009 «Il teatro della vita. Le rappresentazioni dell'etnodiversità», in P. Grimaldi, L. Nattino (a cura di), Il teatro della vita. Le feste tradizionali in Piemonte, Omega, Torino: 7-15.

Grimaldi P., 2010 «Rattoppare la Terra. La ri-nascita del Carnevale di Lajetto e la patrimonializzazione dei beni culturali della tradizione», in D. Porporato (a cura di), Nuove pratiche di comunità. I patrimoni culturali etnoantropologici fra tradizione e complessità sociale, Omega, Torino: 117-171.

Grimaldi P., Porporato D., 2012 Granai della memoria. Manuale di umanità 2.0, Università degli Studi di Scienze Gastronomiche, Bra.

Hurtrel d'Arboval L. H. J., 1839 Dizionario di medicina chirurgia ed igiene veterinaria, vol. 1, Matteo Casali, Forlì.

Lenclud G., 2001 «La tradizione non è più quella di un tempo», in P. Clemente, F. Mugnaini (a cura di), Oltre il folklore. Tradizioni popolari e antropologia nella società contemporanea, Carocci, Roma: 123-134.

Lévi-Strauss C., 1962 Le totemisme aujourd'hui, Puf, Paris; trad. it. 1964 Il totemismo oggi, Feltrinelli, Milano, 1964.

Maffi L., Woodley E., 2010 Biocultural diversity conservation. A Global Sourcebook, Earthscan, LondonNew York.

Manzoni A., 1962 I promessi sposi, La Nuova Italia, Firenze.

Maragliano A., 1962 Tradizioni popolari Vogheresi, a cura di G. Vidossi, I. Maragliano, Le Monnier, Firenze

Milano S. et al., 2015 L'arca del gusto. Come costruire il più grande catalogo dei sapori del mondo: un patrimonio da scoprire e da salvare, Slow Food, Bra. (Testo disponibile in rete, ultimo accesso 8/1/2018, https://www.fondazioneslowfood.com/wp-content/uploads/2015/04/ arca_libretto_ITA.pdf).

Montanari M., 2006 Il cibo come cultura, Laterza, Roma-Bari.

Morris D., 1983 I gesti. Origini e diffusione, Mondadori, Milano. 
Nattino L., 2010 «Festa, comunità, sviluppo», in D. Porporato (a cura di), Nuove pratiche di comunità. I patrimoni culturali etnoantropologici fra tradizione e complessità sociale, Omega, Torino: 259-263.

Peano C., Sottile F. (a cura di), 2012 I Presidi Slow Food in Europa, un modello di sostenibilità. Valutazione dei risultati socioculturali, agroambientali ed economici, Slow Food-Fondazione Slow Food per la Biodiversità Onlus, Bra.

Peano C. et al., 2014 A methodology for the sustainability assessment of agri-food systems: an application to the Slow Food Presidia project. (Testo disponibile in rete, ultimo accesso 1/2/2018, http:// dx.doi.org/10.5751/ES-06972-190424).

Petrini C., 2005a Slow food revolution. Da Arcigola a Terra Madre: una nuova cultura del cibo e della vita, conversazione con Gigi Padovani, Rizzoli, Milano.

Petrini C., 2005b Buono, pulito e giusto. Principi di nuova gastronomia, Einaudi, Torino.

Petrini C., 2009 Terra Madre: come non farci mangiare dal cibo, Giunti, Firenze-Milano; Slow Food, Bra.

Petrini C., 2012 «Il valore delle economie locali e la centralità delle comunità del cibo per una agricoltura buona pulita e giusta», in Rivista di economia agraria, LXVII, n. 3: 11-24.

Petrini C., 2013 Cibo e libertà. Slow Food: storie di gastronomia per la liberazione, Giunti, FirenzeMilano; Slow Food Editore, Bra.

Picchiarelli I., 2004 «Fondazione e rifondazione del tempo e dello spazio», in G. Boirivant, et al. (a cura di), L'antico nella formazione e nel curricolo. Proposte di percorsi didattici per le Scuole superiori di ogni ordine, Franco Angeli, Milano: 349-379.

Plinio G. S., 1982-1988 Storia Naturale, voll. I-V, Einaudi, Torino.

Pouillon J., 1975 Fétiches sans fétichisme, Maspero, Paris.

Porporato D., 2016 «L'etichetta: dall'analogico al digitale / The Label: from analogic to digital» in P. Grimaldi (a cura di), La storia è lunga e bella. Cinquant'anni di Eurostampa / A Long and Beautiful Story. 50 years of Eurostampa, Università di Scienze Gastronomiche, Bra; Eurostampa, Benevagienna: 196-199.

Porporato D., Fassino G., 2016 «I 'Granai della memoria': un'esperienza di patrimonializzazione del territorio tra ricerca e didattica», in G. Germano (a cura di), Per la valorizzazione del patrimonio culturale della Campania. Il contributo degli studi medio- e neo-latini, Loffredo editore, Napoli: 179-193. Raisson V., 20102033 Atlas des futurs du monde, Robert Laffont, Paris; trad. it. 2012 Atlante dei futuri del mondo. Migrazioni, agricoltura, acqua, clima. 2033, Slow Food Editore, Bra.

Rivera A., 1988 Il mago, il santo, la morte, la festa. Forme religiose nella cultura popolare, Dedalo, Bari. Simonetti L., 2010 Mangi chi può: meglio, meno e piano. L'ideologia di Slow Food, Pagliai, Firenze. Siniscalchi V., 2014 «Slow Food Activism between Politics and Economy», in C. Counihan, V. Siniscalchi (eds), Food Activism: Agency, Democracy and Economy, Bloomsbury, London: 225-241.

Teti V., 2004 Il senso dei luoghi. Memoria e storia dei paesi abbandonati, Donzelli, Roma.

Teti V., 2017 Quel che resta. L'Italia dei paesi, tra abbandoni e ritorni, Donzelli, Roma.

Zucca G. D., 1992 «Intorno ai mangiari rituali di Castellazzo e dintorni», in La Ricerca Folklorica, 26: 107-129. 
Foto 1. Morozzo (Cuneo). 2011, Fiera del cappone

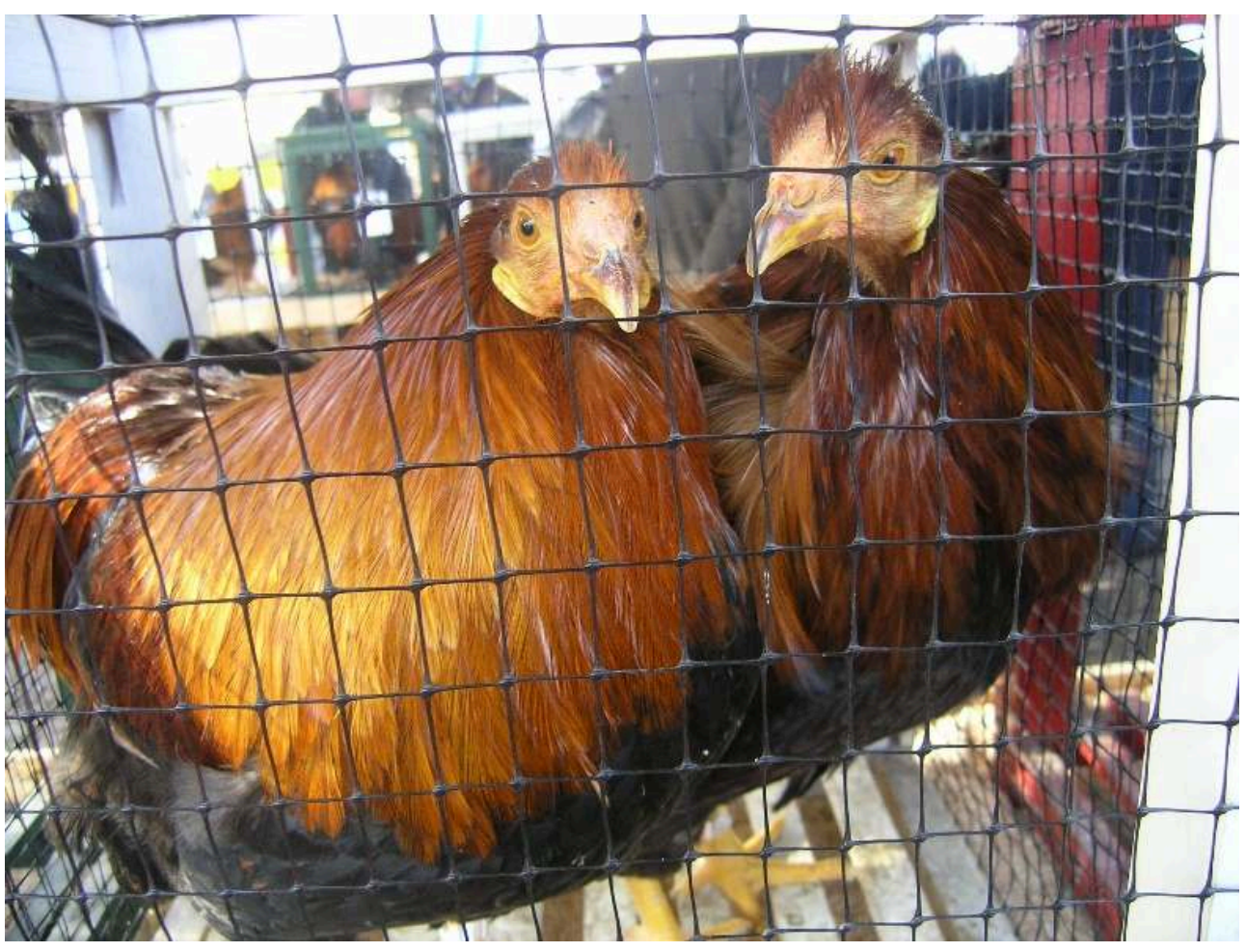

COPPIA DI CAPPONI IN MOSTRA. 
Foto 2. Morozzo (Cuneo). 2011, Fiera del cappone

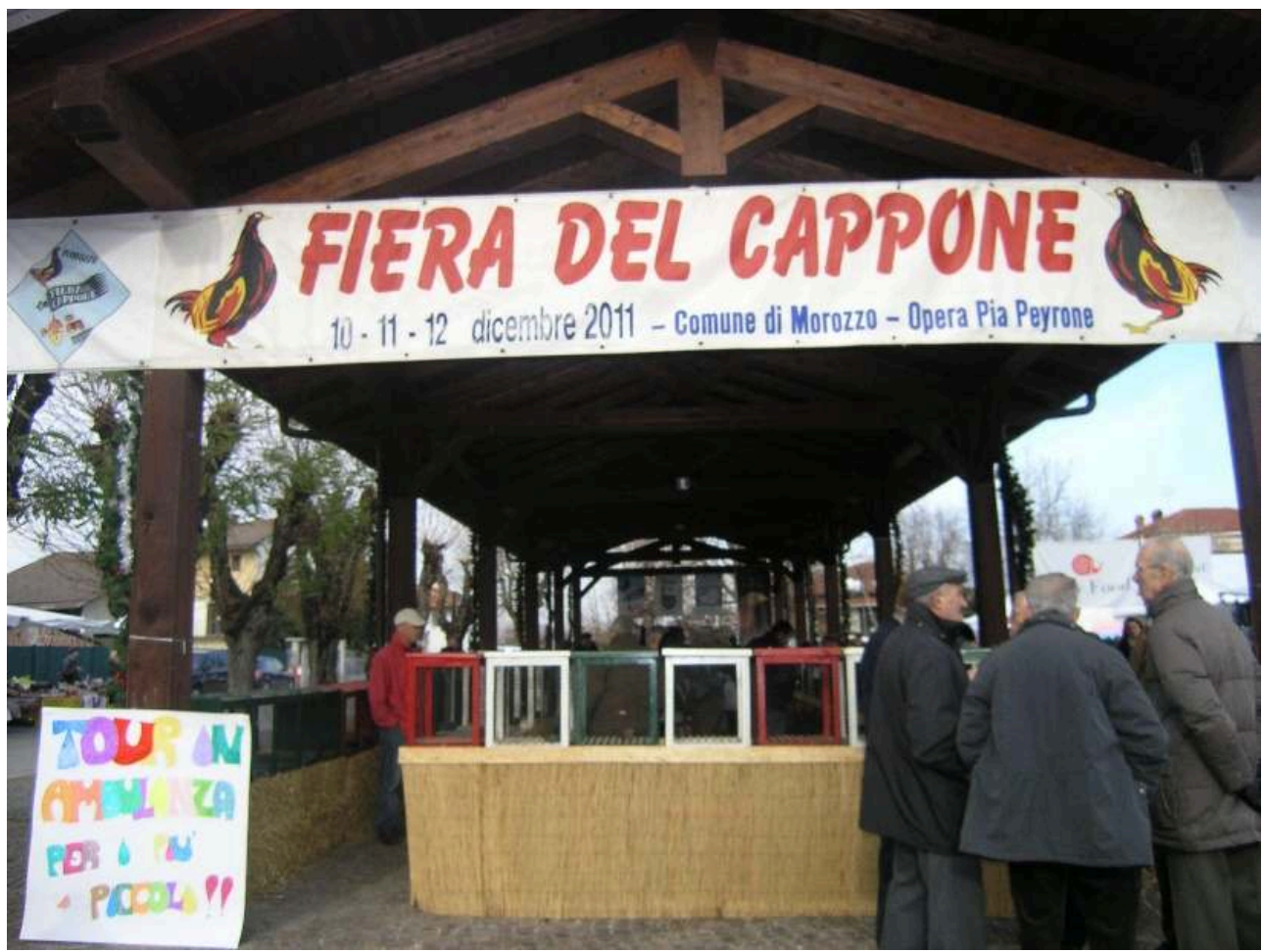

Foto 3. Morozzo (Cuneo)

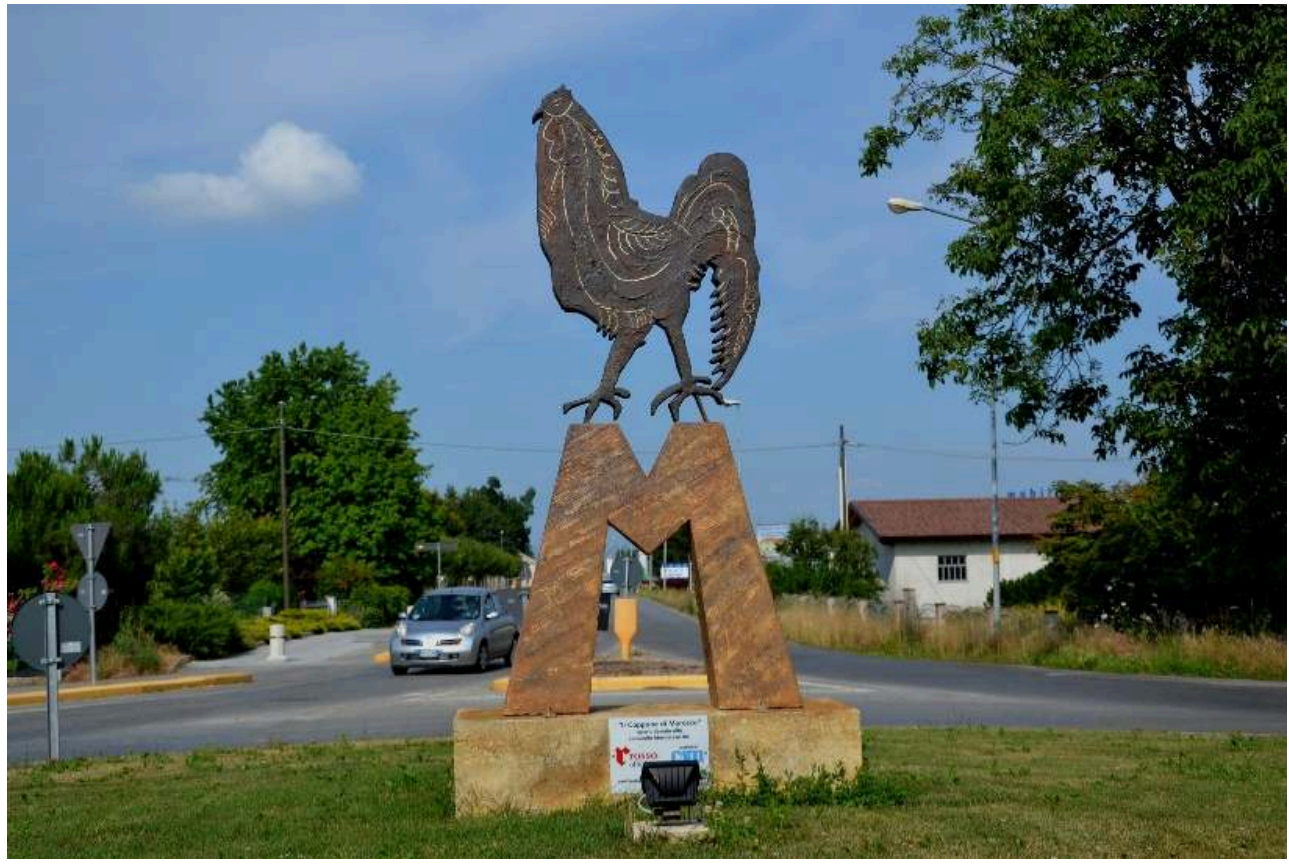

L'IMMAgINE DEL CAPPONE STILIZZATA ALL'INgRESSO DEL PAESE TESTIMONIA LA RITROVA IMPORTANZA DELLA SUA PRODUZIONE. 


\section{NOTE}

1. Si vedano i risultati del Millennium Ecosystem Assessment, una vasta ricerca promossa dalle Nazioni Unite con il fine di valutare le conseguenze dei cambiamenti degli ecosistemi e indicare le azioni necessarie per rendere la vita sul pianeta sostenibile (http:// www.millenniumassessment.org/en/index.html).

2. Si veda: https://www.fondazioneslowfood.com/it/cosa-facciamo/i-presidi/.

3. Associazione nata nel 1989 a seguito dell'esperienza maturata nell'ambito dell'Associazione Gastronomica Arci Gola fondata nel gennaio 1988. Per un approfondimento si vedano: Andrews 2008; Petrini 2005a; Siniscalchi 2014; in una prospettiva critica su Slow Food si vedano Simonetti 2010; Bukowski 2015.

4. Il Manifesto del progetto, redatto nel giugno 1997, si apre con questa premessa: «Per preservare la piccola produzione agroalimentare artigianale di qualità dal diluvio dell'omologazione industriale; per impedire che la velocità divori ed estingua centinaia di razze animali, di salumi di formaggi, di erbe commestibili spontanee o coltivate, di cereali, di frutta; perché cresca l'educazione del gusto; per combattere l'iperigienismo esasperato, che uccide la particolarità di molte produzioni; per tutelare il diritto al piacere. Noi esponenti della cultura, dell'enogastronomia, della ricerca scientifica, del giornalismo, della politica, delle istituzioni, unitamente a quanti vorranno unirsi a noi per raggiungere questi obiettivi, accogliendo e sottoscrivendo il grido di allarme lanciato da slow Food» (https:// www.fondazioneslowfood.com/it/cosa-facciamo/arca-del-gusto/il-progetto/storia/).

5. Vito Teti $(2004 ; 2017)$ chiarisce bene come l'abbandono, lo spopolamento e lo svuotamento dei territori possano diventare occasioni per pensare e comprendere la storia antropologica del nostro Paese. Si apre in questo modo uno spazio d'indagine che opportunamente Teti definisce 'antropologia dell'abbandono', ovvero il tentativo di comprendere l'agire umano nei termini del disconoscimento di sé e della dimenticanza delle proprie origini. Il tema è affrontato anche nella Quarta relazione sulla coesione sociale ed economica della Commissione Europea, nella quale si evidenzia un dato allarmante: un comune italiano su due presenta forme di sofferenza e fenomeni di spopolamento (Teti 2017: 8-9).

6. Nella prospettiva di Slow Food sono tre le direttrici da considerare per definire il concetto di sviluppo sostenibile: «1) la sostenibilità sociale: la capacità di garantire l'accesso a beni considerati fondamentali (sicurezza, salute, istruzione) e a condizioni di benessere (divertimento, serenità, socialità), in modo equo all'interno delle comunità; 2) la sostenibilità ambientale: la capacità di mantenere nel tempo qualità e riproducibilità delle risorse naturali, di preservare la biodiversità e di garantire l'integrità degli ecosistemi; 3) la sostenibilità economica: la capacità di generare in modo duraturo reddito e lavoro e di raggiungere un'eco-efficienza intesa come uso razionale delle risorse disponibili e come riduzione dello sfruttamento delle risorse non rinnovabili» (Peano, Sottile 2012: 9). Tra le numerose pubblicazioni che affrontano il complesso tema della sostenibilità segnaliamo Gianfranco Bologna, 2008. Il volume offre una ricostruzione storica del concetto di sostenibilità in una prospettiva interdisciplinare e presenta un approfondimento sui temi del cambiamento climatico e dell'impoverimento della biodiversità. Si rimanda anche al noto documento delle Nazioni Unite, Report of the World Commission on Environment and Development: Our Common Future (1987), conosciuto come Rapporto Brundtland, che presenta certamente la più conosciuta definizione di sviluppo sostenibile: «lo sviluppo che è in grado di soddisfare i bisogni della generazione presente, senza compromettere la possibilità che le generazioni future riescano a soddisfare i propri bisogni» (http://www.un-documents.net/ wced-ocf.htm).

7. Il $Q R$ code è un codice, nella forma di un crittogramma, composto da stringhe di segni che coprono una matrice quadrata di pochi centimetri e che spesso appare sull'etichetta nella dimensione di 2,5 per 2,5 centimetri. Le smart labels (Label Active) sono un'evoluzione rispetto al 
$Q R$ code in quanto consentono, tra l'altro, di ovviare al problema del segno grafico sull'etichetta: il codice è invisibile ed è inserito nell'etichetta grazie a una tecnica di stampa. Per entrambe le tecnologie i codici sono letti e interpretati da applicazioni installate nei dispositivi portatili, smartphone e tablet, di ultima generazione.

8. A partire dal 2012 sono state effettuate numerose interviste finalizzate a raccogliere la memoria della capponatura in paesi delle provincie di Biella, Cuneo e Torino. In particolare il ciclo produttivo della capponatura è stato documentato con interviste in video di persone che possiedono narrazioni, saperi, forme e pratiche della tradizione e con rilevazioni attraverso la somministrazione di questionari. Le testimonianze in video raccolte tendono a ricostruire la storia di vita, l'autobiografia dell'intervistato; questo non significa registrare l'universo dell'individuo ma può essere considerato tale anche solo un frammento, una parte dell'esistenza del testimone purché rappresenti un tratto paradigmatico, esemplare, del suo essere al mondo (Grimaldi, Porporato 2012: 25).

9. Il quadro di lento e inesorabile declino della 'Fiera del cappone di Morozzo' è anche confermato da Sandro Doglio (1995: 67).

10. Si vedano in particolare le interviste a Giovanni Supertino e Secondina Villosio disponibili sul portale 'Granai della Memoria' - http://www.granaidellamemoria.it/index.php/it.

11. Sul tema del rapporto fra corno e cappone sono suggestive le osservazioni di Salvatore D'Onofrio che sottolinea «l'utilizzazione che si è fatta degli attributi di certi animali, attribuendo loro un ruolo nel sistema di significazione umana» (1997: 137). Si veda anche Morris (1983: 165).

12. Sul nesso tra la funzione di preannunciare il ritorno della luce e di vittima sacrificale di fine anno si veda Picchiarelli 2004: 367-368.

13. Su questi temi si soffermano Giovanni Supertino e Maria Grazia Bussi intervistati in video nel dicembre 2017. I documenti filmici sono parte dell'archivio online del progetto i 'Granai della Memoria' -http://www.granaidellamemoria.it/index.php/it.

Per un approfondimento si vedano anche Giovanni Biroli (1812: 85) e Francesco Gera (1840: 608).

14. Direttiva 2007/43/CE; Decreto Legislativo n. 181 del 2010; Decreto del Ministero della Salute del 4 febbraio 2013.

15. Il progetto 'Granai della Memoria' (http://www.granaidellamemoria.it/index.php/it) è un'iniziativa scientifica, promossa e realizzata dall'Università di Scienze Gastronomiche e da Slow Food, che parte dal presupposto di creare un archivio multimediale, un 'granaio digitale', di facile e democratico accesso, dove i saperi, documentati nella forma orale del racconto, possano trovare riparo. Un lavoro di ricerca ambizioso, forse utopico, che intende contribuire fattivamente a salvare le 'memorie volatili del mondo' che l'uomo ha utilizzato per dialogare con l'altro e che sono parte costitutiva del suo processo evolutivo. Al progetto 'Granai della Memoria' è stato assegnato il premio 'Patrimonio Culturale-Europa Nostra Awards 2016' nella categoria Ricerca. Si tratta del più alto riconoscimento della Commissione Europea nel settore del patrimonio culturale e naturale.

\section{RIASSUNTI}

Il testo propone una riflessione sull'esperienza ventennale dei Presìdi promossi dall'associazione internazionale Slow Food. Un ambizioso progetto avviato per contrastare la perdita della bio ed etno diversità dei territori attraverso la promozione di interventi finalizzati a valorizzare le produzioni locali. A partire da una ricerca etnografica che ha indagato il ciclo produttivo del 
cappone di Morozzo (Cuneo), un prodotto dell'eccellenza gastronomica che negli anni Novanta era prossimo all'estinzione, si è potuto comprendere più compiutamente la funzione svolta dal primo Presidio istituito da Slow Food.

The text offers a reflection on the two decades of experience of the international slow Food association's Presidia project. This ambitious scheme was launched to combat the loss of bio- and ethnodiversity around the world by promoting interventions aimed at adding value to local food products. Starting with the ethnographic research that identified the production cycle for the capon from Morozzo (province of Cuneo), a product of gastronomic excellence that in the 1990s was close to extinction, it is possible to more fully understand the function performed by the first Presidium established by Slow Food.

\title{
INDICE
}

Keywords : Slow Food, presidia, capon, biodiversity, ethnodiversity

Parole chiave : Slow Food, presìdi, cappone, biodiversità, etnodiversità

\author{
AUTORE \\ DAVIDE PORPORATO \\ Dipartimento di Studi Umanistici, Università del Piemonte Orientale \\ davide.porporato[at]uniupo.it
}

